\title{
On Generalized Simple Singular AP-Injective Rings
}

Raida D. Mahammod

raida.1961@uomosul.edu.iq

College of Computer Sciences and Mathematics University of Mosul

\section{Received on: 21/11/2011}

Husam Q. Mohammad

husam_alsabawi@yahoo.com

Accepted on: 15/02/2012

\section{ABSTRACT}

A ring $\mathrm{R}$ is said to be generalized right simple singular AP-injective, if for any maximal essential right ideal $M$ of $R$ and for any $b \in M, b R / b M$ is AP-injective. We shall study the characterization and properties of this class of rings. Some interesting results on these rings are obtained. In particular, conditions under which generalized simple singular AP-injective rings are weakly regular rings, and Von Neumann regular rings.

Key word: AP-injective Rings, weakly continuous rings, socle of R, Von Neumann regular rings.

$$
\begin{aligned}
& \text { حول الحلقات البسيطة المنفردة المعممة وغامرة من النمط AP- }
\end{aligned}
$$

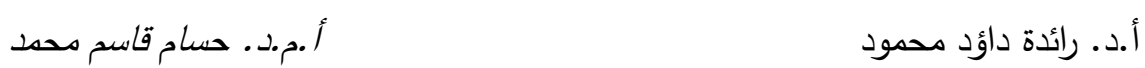

$$
\begin{aligned}
& \text { كلية علوم الحاسوب والرياضيات } \\
& \text { جامعة الموصل }
\end{aligned}
$$

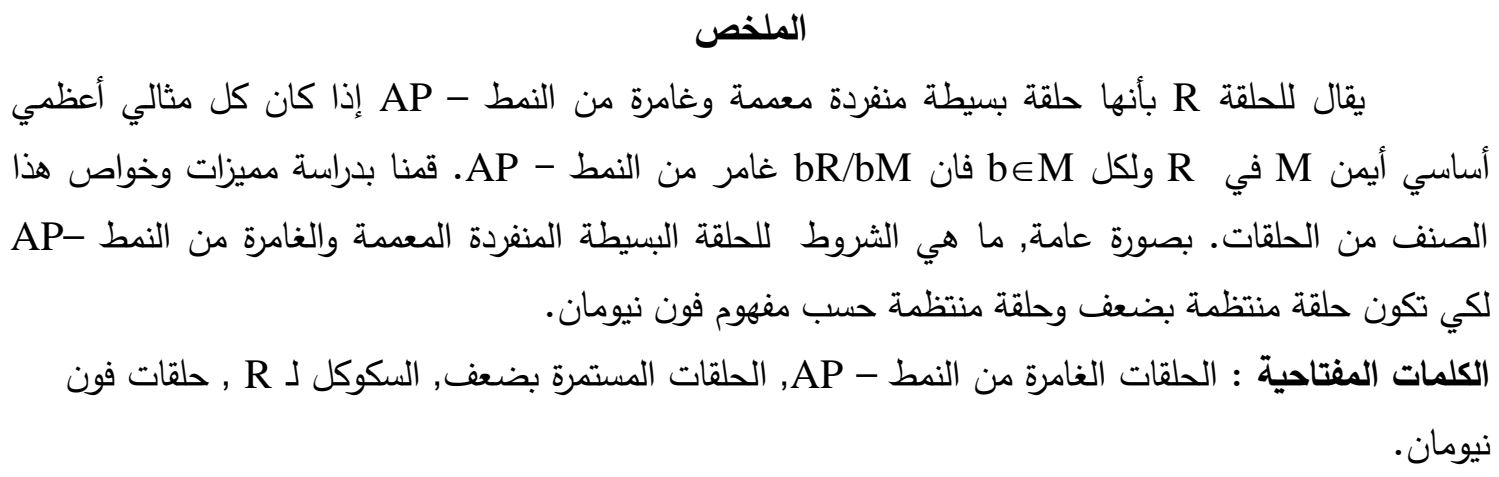

\section{Introduction:}

Throughout this paper, $\mathrm{R}$ is an associative ring with identity, and $\mathrm{R}$-module is unital. For $\mathrm{a} \in \mathrm{R}, \mathrm{r}(\mathrm{a})$ and $\mathrm{l}(\mathrm{a})$ denote the right annihilator and the left annihilator of $\mathrm{a}$, respectively. We write $J(R), Y(R)(Z(R)), N(R)$ and $\operatorname{Soc}\left(R_{R}\right)$ for the Jacobson radical, the right ( left ) singular ideal, the set of nilpotent elements and right socle of $\mathrm{R}$, respectively. $\mathrm{X} \leq \mathrm{M}$ denoted that $\mathrm{X}$ is a submodule of module $\mathrm{M}$.

Recall that a ring $\mathrm{R}$ is called right MC2-ring if $\mathrm{eRa}=0$ implies $\mathrm{aRe}=0$, where $\mathrm{a}$, $\mathrm{e}^{2}=\mathrm{e} \in \mathrm{R}$ and $\mathrm{eR}$ is minimal right ideal of $\mathrm{R}[8]$. A ring $\mathrm{R}$ is Von Neumann (weakly) regular provided that for every $a \in R$ there exists $b \in R(b \in R a R)$ such that $a=a b a(a=a b$ resp.). Recall that a ring $R$ is right (left) weakly continuous if $J(R)=Y(R)(J(R)=Z$ $(R)), R / J(R)$ is regular and idempotent can be left module $J(R)[5]$. Clearly every regular ring is right (left) weakly continuous. A ring $\mathrm{R}$ is called zero commutative (briefly ZC-ring )if $a b=0$ implies $b a=0, a, b \in R[1]$. A right $R$-module $M$ is principally injective (briefly $\mathrm{P}$-injective), if for any principal right ideal aR of $\mathrm{R}$ and any right $\mathrm{R}$ homomorphism of aR into $M$ can be extended to one of $R$ into $M[11]$. The ring $R$ is called right $\mathrm{P}$-injective if $\mathrm{R}_{\mathrm{R}}$ is $\mathrm{P}$-injective.

\section{Generalized Simple Singular AP-injective Rings}


Recall that a module $M_{R}$ with $S=E n d\left(M_{R}\right)$ is said to be almost principally injective (briefly AP-injective), if for any $a \in R$, there exists an S-submodule $X_{a}$ of $M$ such that $\mathrm{l}_{\mathrm{M}}\left(\mathrm{r}_{\mathrm{R}}(\mathrm{a})\right)=\mathrm{Ma} \oplus \mathrm{X}_{\mathrm{a}}$ as left $\mathrm{S}$-module[6]. AP-injectivety has been studied by many authors (see [9,10]). Actually, Zhao Yu-e [12] investigated some properties of rings whose simple singular right $\mathrm{R}$-module is AP-injective. Now, we give a generalized AP-injective.

\section{Definition 2.1:}

A ring $\mathrm{R}$ is called a generalized right (left) simple singular AP-injective, if for any maximal essential right (left) ideal $M$ of $R$, any $b \in M, b R / b M(R b / M b)$ is $A P-$ injective.

The following lemma which is due to Zhao Yu-e [12], plays a central role in several of our proofs

\section{Lemma 2.2:}

Suppose $M$ is a right $R$-module with $S=E n d\left(M_{R}\right)$. If $l_{M r_{R}}(a)=M_{a} \oplus X_{a}$, where $X_{a}$ is left $S$-submodule of $M_{R}$. Set $f: a R \rightarrow M$ is a right $R$-homomorphis, then $f(a)=$ ma+x with $\mathrm{m} \in \mathrm{M}, \mathrm{x} \in \mathrm{X}_{\mathrm{a}}$.

\section{Lemma 2.3:}

$1-\mathrm{aR} \neq \mathrm{aM}$

If $\mathrm{M}$ is a maximal right ideal of $\mathrm{R}$ and $\mathrm{r}(\mathrm{a}) \subseteq \mathrm{M}$ with $\mathrm{a} \in \mathrm{M}$, then

2- $\mathrm{R} / \mathrm{M} \cong \mathrm{aR} / \mathrm{aM}$.

\section{Proof:}

(1) If $\mathrm{aR}=\mathrm{aM}$, then $\mathrm{a}=$ ay for some $\mathrm{y}$ in $\mathrm{M}$, which implies that $1-\mathrm{y} \in \mathrm{r}(\mathrm{a}) \subseteq \mathrm{M}$, whence $1 \in \mathrm{M}$, contradicting $\mathrm{M} \neq \mathrm{R}$.

(2) From (1) $\mathrm{aR} \neq \mathrm{aM}$, then the right $\mathrm{R}$ - homomorphism $\mathrm{g}: \mathrm{R} / \mathrm{M} \rightarrow \mathrm{aR} / \mathrm{aM}$ is defined by $\mathrm{g}(\mathrm{r}+\mathrm{M})=\mathrm{ar}+\mathrm{aM}$ for all $\mathrm{r} \in \mathrm{R}$ implies that $\mathrm{R} / \mathrm{M} \cong \mathrm{aR} / \mathrm{aM}$.

We start this section with the following results.

\section{Proposition 2.4:}

Let $\mathrm{R}$ be generalized right simple singular AP-injective ring, then

$1-\mathrm{J}(\mathrm{R}) \cap \mathrm{Y}(\mathrm{R})=0$

2- $\operatorname{Soc}\left(R_{R}\right) \cap Y(R)=0$

\section{Proof :}

(1) Let $a \in J(R) \cap Y(R)$. If $a \neq 0$, then $r(a) \neq R$ and $R a R+r(a)$ is an essential right ideal of $\mathrm{R}$. We shall prove that $\mathrm{RaR}+\mathrm{r}(\mathrm{a})=\mathrm{R}$. If not, there exists a maximal essential right ideal $M$ containing $R a R+r(a)$. Since $r(a) \subseteq M$ and $a \in M$, then by Lemma $2.3 R / M \cong$ $\mathrm{aR} / \mathrm{aM}$. Therefore, $\mathrm{R} / \mathrm{M}$ is AP-injective and $\mathrm{l}_{\mathrm{R} / \mathrm{Mr}} \mathrm{r}(\mathrm{a})=(\mathrm{R} / \mathrm{M}) \mathrm{a} \oplus \mathrm{X}_{\mathrm{a}}, \mathrm{X}_{\mathrm{a}} \leq \mathrm{R} / \mathrm{M}$. Let $\mathrm{f}: \mathrm{aR} \rightarrow \mathrm{R} / \mathrm{M}$ defined by $\mathrm{f}(\mathrm{ar})=\mathrm{r}+\mathrm{M}$ for all $\mathrm{r} \in \mathrm{R}$. Note that $\mathrm{f}$ is a well-defined and by Lemma $2.21+\mathrm{M}=\mathrm{f}(\mathrm{a})=\mathrm{ba}+\mathrm{M}+\mathrm{x}, \mathrm{b} \in \mathrm{R}, \mathrm{x} \in \mathrm{X}_{\mathrm{a}}$. Hence $1-\mathrm{ba}+\mathrm{M}=\mathrm{x} \in \mathrm{R} / \mathrm{M}$ $\cap X_{a}=0$, so $1-b a \in M$. Since $a \in J(R)$, then $b a \in J(R) \subseteq M$ and hence $1 \in M$, which is a contradiction. Therefore $\mathrm{J}(\mathrm{R}) \cap \mathrm{Y}(\mathrm{R})=0$.

(2) Let $k \in \operatorname{Soc}\left(R_{R}\right) \cap Y(R)$. If $k \neq 0$, then $k R$ is a minimal right ideal and $r(k)$ is an essential right ideal of $\mathrm{R}$. Since every minimal one -sided ideal of $\mathrm{R}$ is either nilpotent or direct summand of $\mathrm{R}$ [8]. Thus, if $(\mathrm{kR})^{2} \neq 0$, then $\mathrm{kR}$ is a direct summand and hence $\mathrm{r}(\mathrm{k})$ is also direct summand which is a contradiction. If 
$(\mathrm{kR})^{2}=0$, then $\mathrm{k}^{2}=0$ and $\mathrm{k} \in \mathrm{r}(\mathrm{k})$. But $\mathrm{r}(\mathrm{k})$ is maximal essential right ideal of $\mathrm{R}$. Therefore, by Lemma $2.3 \mathrm{R} / \mathrm{r}(\mathrm{k}) \cong \mathrm{k}(\mathrm{r}(\mathrm{k}))$. Hence, $\mathrm{R} / \mathrm{r}(\mathrm{k})$ is AP-injective, so there exists $c \in R$ and $x \in X_{a}$ as a proof (1) such that $1-c k \in r(k)$. Since, ck $\in R k R \subseteq r(k)$, then $1 \in \mathrm{r}(\mathrm{k})$. This is also contradiction, therefore $\operatorname{Soc}\left(\mathrm{R}_{\mathrm{R}}\right) \cap \mathrm{Y}(\mathrm{R})=0$.

Following [7], for a prime ideal $\mathrm{P}$ of a ring $\mathrm{R}$, we put $\mathrm{O}_{\mathrm{p}}=\{\mathrm{a} \in \mathrm{P}: \mathrm{ab}=0$ for some $\mathrm{b} \in \mathrm{R} \backslash \mathrm{P}\}$. In general, $\mathrm{O}_{\mathrm{P}}$ not subset of a prime ideal $\mathrm{P}$. as the following example shows.

\section{Example [2]:}

Let $\mathrm{R}$ be a ring of $2 \times 2$ matrices over a field $\mathrm{F}$. Then, $\mathrm{P}=\left[\begin{array}{ll}0 & 0 \\ 0 & 0\end{array}\right]$ is a prime ideal of $R$. Let $a=\left[\begin{array}{ll}0 & 0 \\ 1 & 0\end{array}\right], b=\left[\begin{array}{ll}0 & 0 \\ 0 & 1\end{array}\right], \in R$. Then $a b=\left[\begin{array}{ll}0 & 0 \\ 0 & 0\end{array}\right]$ and $b \in R \backslash P$. Thus, $a \in O_{P}$, but. $\mathrm{a} \notin \mathrm{P}$

\section{Theorem 2.5:}

Let $\mathrm{P}$ be a prime ideal of a generalized right simple singular AP-injective ring with $\mathrm{O}_{\mathrm{P}} \subseteq \mathrm{P}$, then $\mathrm{P}$ is maximal.

\section{Proof :}

We claim that $\mathrm{RaR}+\mathrm{P}=\mathrm{R}$ for $\mathrm{a} \in \mathrm{R} / \mathrm{P}$. if not, there exists a maximal ideal $\mathrm{M}$ of $\mathrm{R}$ containing $\mathrm{RaR}+\mathrm{P}$. Moreover, $\mathrm{M}$ is a maximal right ideal of $\mathrm{R}$. Suppose not, then there exists a maximal right ideal $\mathrm{K}$ of $\mathrm{R}$ such that $\mathrm{M} \subseteq \mathrm{K}$. If $\mathrm{K}$ is not essential in $\mathrm{R}$. Then $K$ is a direct summand of $R$, so we can write $K=r(e)$ for some $0 \neq e=e^{2} \in R$. Then, ea $=0$, since $e \notin P$, then $a \in O_{P} \subseteq P$. Therefore, $K$ must be essential right ideal of $R$.

Now, suppose that $a R=a K$, then $a=a c$ for some $c \in K$ that implies $a(1-c)=0$. Since, $\mathrm{a} \notin \mathrm{P}$, then $1-\mathrm{c} \in \mathrm{O}_{\mathrm{P}} \subseteq \mathrm{P} \subseteq \mathrm{K}$ which is a contradiction. If $\mathrm{aR} \neq \mathrm{aK}$, the right $\mathrm{R}$ homomorphism $g: R / K \rightarrow a R / a K$ is defined by $g(b+K)=a b+a K$ for all $b \in R$ which implies that $R / K \cong a R / a k$. Therefore, $R / K$ is $A P$-injective . Let $f: a R \rightarrow R / K$ be defined by $f(a r)=r+K$ for all $r \in R$. So by Lemma $2.2 f(a)=c a+K+x, x \in X_{a}$. Hence, 1$c a+K=x \in R / K \square X_{a}=0$, so $1-c a \in K$ whence $1 \in K$. Therefore, $M$ is a maximal essential right ideal of $\mathrm{R}$. So by the same method in the above proof $\mathrm{P}$ is a maximal of $\mathrm{R}$.

Recall that $R$ is called 2-Primal if its prime radical $P(R)$ concedes with the set $N(R)$ [7]. Kim and Kwak [3] showed that if $R$ is a 2-primal, then $\mathrm{OP}_{\mathrm{P}} \subseteq \mathrm{P}$ for each prime ideal of $\mathrm{R}$.

\section{Corollary 2.6:}

Let $\mathrm{R}$ be 2-primal generalized right simple singular AP-injective ring, then every prime ideal of $\mathrm{R}$ is maximal.

\section{Proposition 2.7:}

Let $\mathrm{R}$ be $\mathrm{ZC}$-generalized simple singular AP-injective rings, then for any $\mathrm{a}, \mathrm{b} \in$ $R$ with $a b=0$, then $r(a)+r(b)=R$.

\section{Proof:}

Suppose that $a b=0$ and $r(a)+r(b) \neq R$. Then, there exists a maximal right ideal $M$ containing $r(a)+r(b)$. If $M$ not essential, then there exists $0 \neq \mathrm{e}=\mathrm{e}^{2} \in \mathrm{R}$ such that $M=r(e)$. Since $b \in r(a) \subseteq M=r(e)=l(e)$, then be=0 which implies that $e \in r(b) \subseteq M=r(e)$, so that $\mathrm{e}=\mathrm{e}^{2}=0$ which is a contradiction. Therefore, $M$ must be essential.

Since, $\mathrm{r}(\mathrm{a}) \subseteq \mathrm{M}$ and $\mathrm{a} \in \mathrm{M}$, then by Lemma $2.3 \mathrm{R} / \mathrm{M} \cong \mathrm{aR} / \mathrm{aM}$. Therefore $\mathrm{R} /$ $M$ is AP-injective. Let $f: a R \rightarrow R / M$ is defined by $f($ ar $)=r+M$ for all $r \in R$. Note that $f$ is 
well-defined and by Lemma $2.21+\mathrm{M}=\mathrm{f}(\mathrm{a})=\mathrm{ca}+\mathrm{M}+\mathrm{x}, \mathrm{c} \in \mathrm{R}, \mathrm{x} \in \mathrm{X}_{\mathrm{a}}$. Hence, $1-$ $\mathrm{ca}+\mathrm{M}=\mathrm{x} \in \mathrm{R} / \mathrm{M} \cap \mathrm{X}_{\mathrm{a}}=0$, so 1 -ca $\in$ M. Since, $\mathrm{a} \in \mathrm{r}(\mathrm{b})$ and $\mathrm{R}$ is $\mathrm{ZC}$ - ring, then ca $\in$ $\mathrm{r}(\mathrm{b}) \subseteq \mathrm{M}$ whence $1 \in \mathrm{M}$ which is a contradiction. Therefore, $\mathrm{r}(\mathrm{a})+\mathrm{r}(\mathrm{b})=\mathrm{R}$.

\section{The Connection between Generalized Simple Singular AP-injective and Other Rings}

In this section, we give the connection between Von Neumann regular rings and generalized simple singular AP-injective rings.

\section{Theorem 3.1:}

Let $\mathrm{R}$ be right $\mathrm{MC2}$-generalized right simple singular AP-injective, then $\mathrm{R}$ is right weakly regular ring.

\section{Proof:}

We will show that $R a R+r(a)=R$ for any $a \in R$. Suppose that there exists $b \in R$ such that $\mathrm{RbR}+\mathrm{r}(\mathrm{b}) \neq \mathrm{R}$. Then, there exists a maximal right ideal $\mathrm{M}$ of $\mathrm{R}$ containing $\mathrm{RbR}+\mathrm{r}(\mathrm{b})$. If $\mathrm{M}$ not essential, then $\mathrm{M}$ is a direct summand of $\mathrm{R}$. So, we can write $M=e R$ for some $0 \neq e=e^{2} \in R$. Thus, (1-e)Rb=0, since $R$ is MC2 and (1-e)R is minimal, then $\mathrm{bR}(1-\mathrm{e})=0$. Hence, $(1-\mathrm{e}) \in \mathrm{r}(\mathrm{b}) \subseteq \mathrm{M}$, so $1 \in \mathrm{M}$. It is a contradiction. Therefore, $\mathrm{M}$ must be essential right ideal of $\mathrm{R}$.

Since, $r(a) \subseteq M$ and $a \in M$, then by Lemma $2.3 \mathrm{R} / \mathrm{M} \cong \mathrm{aR} / \mathrm{aM}$. Therefore, $\mathrm{R} /$ $M$ is AP-injective. Let $f: b R \rightarrow R / M$ defined by $f(b r)=r+M$ for all $r \in R$. Note that $f$ is well-defined and by Lemma 2.2, $1+\mathrm{M}=\mathrm{f}(\mathrm{b})=\mathrm{cb}+\mathrm{M}+\mathrm{x}, \mathrm{c} \in \mathrm{R}, \mathrm{x} \in \mathrm{X}_{\mathrm{b}}$. Hence, $1-\mathrm{cb}$ $+M=x \in R / M \cap X_{b}=0$, so $1-c b \in M$. Since, $c b \in R b R \subseteq M$, then $1 \in M$ which is a contradiction. Therefore, that $\mathrm{RaR}+\mathrm{r}(\mathrm{a})=\mathrm{R}$ for all $\mathrm{a} \in \mathrm{R}$. Hence, $\mathrm{R}$ is a right weakly regular ring.

Now, we shall prove the main results of this section.

\section{Theorem 3.2:}

Let $\mathrm{R}$ be a ring, then the following statements are equivalent:

(1) $R$ is Von Neumann regular.

(2) $\mathrm{R}$ is generalized right simple singular AP-injective right weakly continuous.

\section{Proof :}

$(1) \Rightarrow(2)$ It is clear.

(2) $\Rightarrow$ (1) Suppose that $Y(R) \neq 0$. Then, there exists a non-zero element a $\in Y(R)$ such that $\mathrm{a}^{2}=0$. We claim that $\mathrm{Y}(\mathrm{R})+\mathrm{r}(\mathrm{a})=\mathrm{R}$. If not, there exists a maximal essential right ideal $M$ containing $Y(R)+r(a)$. Since, $r(a) \subseteq M$ and $a \in M$, then by Lemma $2.3 R / M \cong$ $\mathrm{aR} / \mathrm{aM}$. Therefore, R/M is AP-injective and $\mathrm{l}_{\mathrm{R} / \mathrm{M}} \mathrm{r}(\mathrm{a})=(\mathrm{R} / \mathrm{M}) \mathrm{a} \oplus \mathrm{X}_{\mathrm{a}}, \mathrm{X}_{\mathrm{a}} \leq \mathrm{R} / \mathrm{M}$. Let $\mathrm{f}: \mathrm{aR} \rightarrow \mathrm{R} / \mathrm{M}$ be defined by $\mathrm{f}($ ar $)=\mathrm{r}+\mathrm{M}$ for all $\mathrm{r} \in \mathrm{R}$. Note that $\mathrm{f}$ is well-defined and by Lemma 2.2, $1+M=f(a)=b a+M+x, b \in R, x \in X_{a}$. Hence, $1-b a+M=x \in R / M$ $\cap X_{a}=0$, so 1 -ba $\in M$. Since, $a \in Y(R)=J(R)$ implies that $c a \in J(R) \subseteq M$ and $1 \in M$, which is a contradiction. Therefore, $Y(R)+r(a)=R$. Thus, we can write $1=c+d$, for some $c \in Y(R)$ and $d \in r(a)$. Thus, $a=c a$ and so $(1-c) a=0$. Since $c \in Y(R)=J(R), 1-c$ is invertible. Thus $\mathrm{a}=0$ contradicting $\mathrm{a} \neq 0$. Therefore, $\mathrm{Y}(\mathrm{R})=0$.

\section{Lemma 3.3: [4]}

For any $a \in \operatorname{Cent}(R)$, if $a=$ ara for some $r \in R$, then there exists $b \in \operatorname{Cent}(R)$ such that $a=a b a$ ( where $\operatorname{Cent}(R)$ is the center of $R$ ).

\section{Theorem 3.4:}


$\mathrm{R}$ is right non-singular generalized right simple singular AP-injective, then Cent(R) is Von Neumann regular ring.

\section{Proof:}

First, we have to prove $\operatorname{Cent}(R)$ is reduced . Let $0 \neq a \in \operatorname{Cent}(R)$ and $a^{2}=0$ implies that $a \in r(a)$.If $r(a)$ is essential, then $a \in Y(R)=0$ implies that $a=0$.We are done . If $r(a)$ not essential ,there exists a non-zero right ideal $I$ in $R$ such that $r(a) \cap I=0$.Then, $\mathrm{I} \subseteq \mathrm{I} \cap \mathrm{r}(\mathrm{a})[\mathrm{a} \in \mathrm{Cent}(\mathrm{R})]$ but $\mathrm{I} \cap \mathrm{r}(\mathrm{a})=0$ implies that $\mathrm{I} a=0$ and we get $\mathrm{I} \subseteq \mathrm{l}(\mathrm{a})=\mathrm{r}(\mathrm{a})$ so $\mathrm{I}=0$ contradiction. Therefore, $a=0$,so $\operatorname{Cent}(R)$ is a reduced ring . Now, we shall show that $a R+r(a)=R$ for any $a \in \operatorname{Cent}(R)$.If not ,there exists a maximal right ideal $M$ of $R$ such that $\mathrm{aR}+\mathrm{r}(\mathrm{a}) \subseteq \mathrm{M}$ observe that $\mathrm{M}$ is an essential right ideal of $\mathrm{R}$. If not, then $\mathrm{M}$ is a direct summamd of $R$. So, we can write $M=r(e)$ for some $0 \neq e=e^{2} \in R$. Since, $a \in M$ and $a \in$ $\operatorname{Cent}(\mathrm{R}), \mathrm{ae}=\mathrm{ea}=0$. Thus, $\mathrm{e} \in \mathrm{r}(\mathrm{a}) \subseteq \mathrm{M}=\mathrm{r}(\mathrm{e})$, whence $\mathrm{e}=0$. It is a contradiction. Therefore, $M$ must be an essential right ideal of $R$.

Since, $\mathrm{r}(\mathrm{a}) \subseteq \mathrm{M}$ and $\mathrm{a} \in \mathrm{M}$, then by Lemma $2.3 \mathrm{R} / \mathrm{M} \cong \mathrm{aR} / \mathrm{aM}$. Therefore, $\mathrm{R} /$ $\mathrm{M}$ is AP-injective. Let $\mathrm{f}: \mathrm{aR} \rightarrow \mathrm{R} / \mathrm{M}$ defined by $\mathrm{f}($ ar $)=\mathrm{r}+\mathrm{M}$ for all $\mathrm{r} \in \mathrm{R}$. Note that $\mathrm{f}$ is well-defined and by Lemma 2.2, $1+\mathrm{M}=\mathrm{f}(\mathrm{a})=\mathrm{ca}+\mathrm{M}+\mathrm{x}, \mathrm{c} \in \mathrm{R}, \mathrm{x} \in \mathrm{X}_{\mathrm{a}}$. Hence, $1-$ $c a+M=x \in R / M \cap X_{a}=0$, so 1-ca $\in M$ since, $a \in \operatorname{cent}(R)$, then ca $=a c \in M$, and hence $1 \in \mathrm{M}$. Therefore, $\mathrm{aR}+\mathrm{r}(\mathrm{a})=\mathrm{R}$ for all $\mathrm{a} \in \operatorname{cent}(\mathrm{R})$ and so we have $\mathrm{a}=$ ara for some $\mathrm{r} \in \mathrm{R}$. Applying Lemma 3.3, Cent( $\mathrm{R})$ is Von Neumann regular ring.

\section{REEFERENCES}

[1] Cohn, P.M. (1999), "Reversible Rings“, Bull. London Math. Soc., Vol. 31, pp. 641-648

[2] Hong, C.Y., Kim, N.K. and Kwak, T.K. (2000), "On Rings Whose Prime Ideals are Maximal", Bull. Korean Math. Soc. Vol. 37, No. 1, pp 1-9.

[3] Kim, N.K. and Kwak, T.K., (1999), "Minimal Prime Ideals in 2-Primal Rings", Math. Japonica Vol. 50, No. 3, pp 415-420.

[4] Nam, S.B. and Kim, J.Y. (1999), "A Note On Simple Singular GP-Injective Modules “, Kangweon-Kyungki Math. Jour. Vol. 7, No. 2, pp 215-218.

[5] Nicholson, W.K. and Yousif M.F. (2004), "Quasi-Frobenius Rings“, Cambridge Tracts In Mathematics, Cambridge University Press, Cambridge.

[6] Page S.S., Zhou, Y.Q. (1998), "Generalizations of Principally Injective Rings", J. Algebra, Vol. 206, pp. 706-721.

[7] Shin, G.Y. (1973), "Prime Ideals and Sheaf Representation of a Pseudo Symmetric Ring", Trans. Amer. Soc. Vol. 184, pp43-60.

[8] Wei, J.C. (2005), "The Rings Characterized By Minimal Left Ideal", Acta Mathematica Sinica, English Series 21 (3), pp. 473-482.

[9] Xiao G.S., Ding N.Q. and Tong W.T., (2004), "Regularity of AP-injective rings", Vietnam Journal of Mechanics, Vol. 32, No.4, pp.399-411.

[10] Xiao, G.S., Tong, W.T., (2006), "On Injectivity and P-injectivity", Bull. Korean Math. Soc., Vol. 43, No.2, pp. 299-307. 
[11] Yue Chi Ming, R. (1974), “On Von Neumann Regular Rings“،, Proc. Edinburgh Math. Soc. 19, pp. 89-91.

[12] Zhao-Yu-e, (2011), "On Simple Singular AP-injective modules", International Mathematical Forum, Vol. 6, 2011, No. 21, pp. 1037-1043. 